\title{
Effect of whole body vibration on bone mineral density in children with myelomeningocele
}

\author{
Hatem A.Emara \\ Lecturer in the Department of Physical Therapy for Growth and Developmental Disorders in Children and its \\ Surgery, Faculty of Physical Therapy, Cairo University, Egypt
}

\begin{abstract}
In patients with myelomeningocele (MMC), a high number of fractures occur in the paralyzed extremities, affecting mobility and independence. The purpose of this study was to investigate the effect of whole body vibration (WBV) on bone mineral density in children with MMC. Methodology:20 children with MMC, from both sexes, participated in this study. Their age ranged from 2 to 5 years. They were divided randomly into 2 groups of equal number: Control group (group A), this group received a specially designed physical therapy program for 3 months, 3 times weekly and group B( study group), this group received the same exercise program given to group $A$ in addition to WBV. Bone mineral density (BMD) of the legs, femur and spine was measured using lunar prodigy advance apparatus pre and after 3 months. Results: There was significant increase in BMD in both groups with more improvement in the study group. Conclusion: WBV can be used as an effective method for increasing BMD for children with MMC.
\end{abstract}

Keywords: Bone mineral density, Myelomeningocele, whole body vibration.

\section{Introduction}

Spina bifida is the most frequent of congenital anomalies of the central nervous system that are compatible with life. The most common form of spina bifida is myelomeningocele, characterized by the extrusion of the spinal cord into a sac filled with cerebrospinal fluid, resulting in permanent disability. The incidence of MMC has stabilized at 3.4 per 10,000 live births in the United States, despite folic acid fortification [1]. Long-term survivors have major disabilities, including paralysis and bowel and bladder dysfunction. Damage to the spinal cord and peripheral nerves usually is obvious at birth and is permanent despite early postnatal surgical repair. The level of the injury to the spinal cord determines the severity of the neurologic disability in the lower extremities [2].

The World Health Organization (WHO) has defined osteoporosis as a skeletal disease characterized by "low bone density and microarchitectural deterioration of bone tissue with a consequent increase in bone fragility and susceptibility to fracture" [3].A study by Valtonene et al.[4] was conducted to assess the prevalence of osteoprosis and osteopenia in adults with MMC and to explore whether neurologic level, ambulatory status, and other medical problems are associated with BMD. BMD in the lumbar spine, hip, and forearm were measured with dual X-ray absorptiometry, results showed that $33 \%$, of subjects had osteoprosis in at least 1 of the measured sites, $47 \%$ had osteoprosis in the femoral neck or trochanteric regions of the hip. Subjects with other medical problems frequently occurring in MMC had lower BMD in the femoral neck and trochanteric region of the hip than subjects without such factors. Ambulation alone showed only a tendency to be associated with BMD of the femoral neck, while the effect of other medical risk factors on BMD of the femoral neck was stronger among the nonambulators than the ambulators, the study concluded that osteoprosis is a medical problem to be considered when treating and rehabilitating patients with MMC .

Children with spina bifida cystica have a high risk of fracturing their paraplegic legs. During the last fifteen years, 261 fractures and epiphysis injuries in 137 children out of 1,400 (12.2\%) patients with spina bifida was observed. The increased risk of fracture seems to be due to decreased muscle activity in the paralysed limb with inadequate axial loading of the legs. A large proportion of fractures occurs after orthopedic interventions. Fractures are less frequent after urologic or neurosurgical procedures. Fractures are extremely frequent after operations in association with cast immobilization. Early standing and short immobilization times are the best defense mechanisms against fractures. If plasters are needed postoperatively one should preferably use the "Max and Moritz" standing cast. Fractures ,in spina bifida children, heal quickly as compared to those in non-paralyzed children. Immobilization for fracture care can be done in a standing cast even in the very early phase of treatment. In spite of the swelling and elevated temperature, axial loading in the immobilization for fracture care can be done in a standing cast even in the very early phase of treatment. Axial loading in the standing cast should continue, in spite of the high temperature and swelling [5]. 
In most WBV research studies, subjects stand on a platform or plate that produces sinusoidal oscillations either in a vertical up and down motion or that rotates about a center fulcrum producing up and down vertical motion on alternating sides of the fulcrum. These movements result in vibrations transmitted indirectly to the subject through the legs [6].

The recent research has shown that WBV can be used as an alternative method for stimulating both improvements in muscle performance and increases in bone mass [7-12]. Studies performed on animal have shown that high-frequency, low-magnitude vibration can increase bone density, bone strength, and prevent bone loss [13, 14]. Studies in humans have also shown a benefit to bone in postmenopausal women[7] and a benefit to both muscle and bone in young women, ages 15 to 20 years, with low bone density[9]. In disabled children, a small pilot study found that 6 months of low-magnitude, high-frequency WBV increased bone mass and prevented bone loss in the proximal tibias of a heterogeneous group of participants [10].However, to our knowledge, no previous study has addressed the effect of WBV on BMD in spina bifida children, so the purpose of this study was to examine the effect of high-frequency, low-magnitude WBV on BMD in children with MMC.

\section{Patients, Instrumentation and Procedures}

\subsection{Patients:}

Twenty children from both sexes were selected from the outpatient clinic of Faculty of Physical Therapy, Cairo University(Fig.1),their age ranged from 2 to 5 years .They were referred as having MMC at the lumbosacral level. They were excluded: if they had treatment with bisphosphonate medication, a history of a spine fusion or other procedure resulting in the presence of orthopedic hardware, or a recent history $(<3$ months) of casting of one or more extremity. Children were also excluded if they had a hip flexion contracture greater than 30degrees in both lower extremities or a recent proximal femur fracture, as this prevented an accurate measurement at the femoral neck. Informed consent was obtained from all parents. This study was approved by the Faculty of Physical Therapy, Cairo University. Children were assigned randomly into 2 groups of equal numbers as control group (group A) and study group (group B).

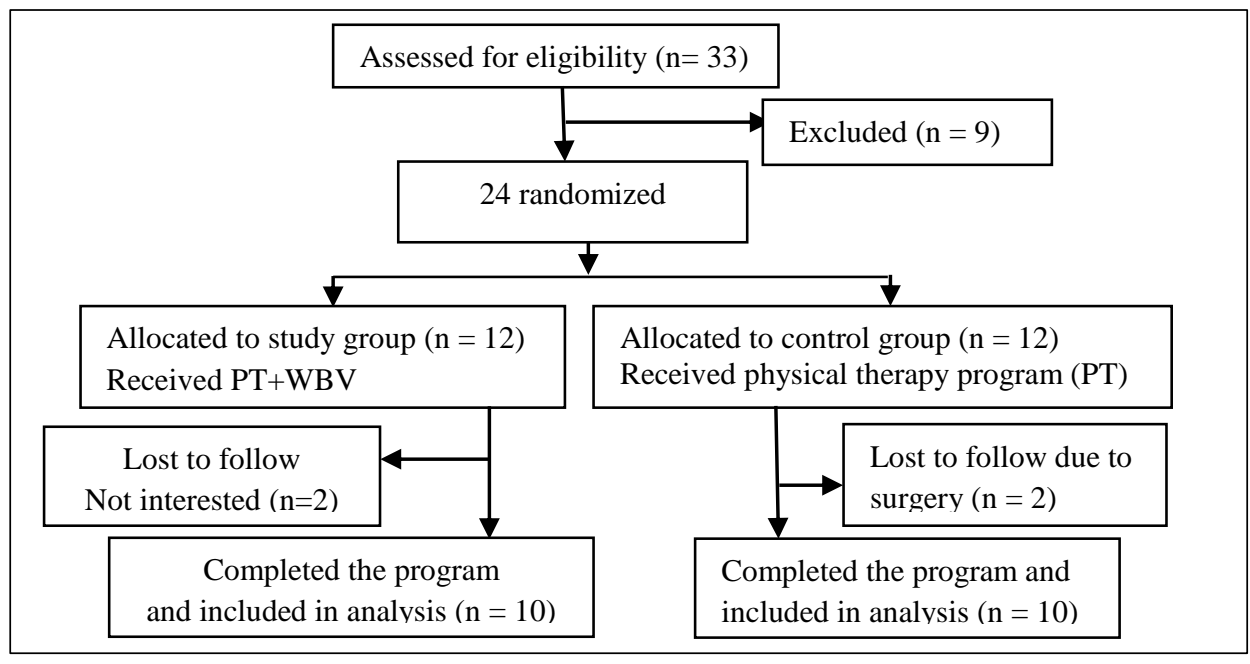

Figure (1): flow chart of participation throughout the study

\subsection{Instrumentation}

2.2.1. Whole body vibration: For the WBV treatment, a commercially available WBV device was used (Vmax Fitness i25).It has console (screen), support tube, vibration plate, base board, right and left hand rails tube.

2.2.2. Mats, wedges, balls, standing frame, rolls and standing bars for conducting the therapeutic exercise program.

\subsection{Procedures:}

2.3.1. Procedures for evaluation:

BMD of the leg (proximal tibia), femoral neck and spine was measured using dual energy X-ray absorptiometry (DEXA; Lunar Corporation, Model DP3, Madison, WI, USA).All children were assessed by DEXA before and after the treatment program .Measurement of BMD passed through the following steps:1-Recording of the child's data,2Selecting the measurement site and 3-Analysis of the measurement. A certified physician interpreted the test data to ensure the consistency of the DEXA quality.

2.3.2. Procedures for treatment:

2.3.2.1.The control group (group A):children in this group received a selected physical therapy program for one hour,3 times weekly. This program included:(1) Facilitation of muscle contraction by tapping, squeezing and quick 
stretch followed by passive movement, (2)Back and abdominal exercises ,(3)Facilitation of postural mechanism and (4)Standing on the standing frame.

2.3.2.2. The study group: Children in this group received the same program given to the control group in addition to whole body vibration. The WBV treatment was administered 3 times weekly by the researcher. The treatment schedule was adapted from published observational studies that had used the same WBV system as the present study to treat children with neuromuscular diseases and bone fragility disorders [16, 17]. Each WBV session consisted of the following schedule :( $3 \mathrm{~min}$ of WBV) - (3 min rest)-(3 min of WBV) - (3 min rest)-(3 min of WBV).During the first 3 min of WBV, child sat on the platform of the WBV device and he was supported by the mother or by the physical therapist. During the second 3 min of WBV, the child was positioned in the kneeling position with $15^{\circ}$ knee flexion holding on the supporting rails. During the last $3 \mathrm{~min}$ of WBV child wore the hip knee immobilizer and stood on the vibration platform holding on the supporting hand rails. Thus, one treatment session corresponded to 9 min of exposure to WBV. All exercises were performed with a pad provided by the manufacturer. The vibration platform operated at $30 \mathrm{~Hz}$ with a peak acceleration of $0.3 \mathrm{~g}[11]$.

\section{Results}

The collected data from this study represent the statistical analysis of the BMD including the leg, the femoral neck and the spine that was measured before and after three months of treatment for the two groups. The raw data of the measured variables for the two groups were statistically treated to show the mean and standard deviation. Student t-test was then applied to examine the significance of the treatment conducted for each group. The obtained results in this study revealed no significant differences when comparing the pretreatment mean values of the two groups. Significant improvement was observed in all the measuring variables of the two groups, when comparing their pre and post-treatment mean values (Table1and Fig.2). After the treatment program significant difference was observed when comparing the post-treatment results of the two groups in favor of the study group.

Table1: The pre and post treatment mean values of the measured variables in both groups

\begin{tabular}{|l|l|l|l|l|l|}
\hline \multirow{2}{*}{ BMD $\left(\mathbf{g m} / \mathbf{c m}^{2}\right)$} & Group & \multicolumn{2}{|c|}{ Mean \pm SD } & t-value & p-value \\
\cline { 3 - 6 } & & Pre & Post & & \\
\hline \multirow{2}{*}{ Leg( proximal tibia) } & Control & $0.319 \pm 0.011$ & $0.366 \pm 0.016$ & 11.57 & $<0.05^{*}$ \\
\cline { 2 - 6 } & Study & $0.325 \pm 0.014$ & $0.441 \pm 0.028$ & 14.44 & $<0.05^{*}$ \\
\hline \multirow{2}{*}{ Neck of femur } & Control & $0.378 \pm 0.008$ & $0.423 \pm 0.008$ & 17.61 & $<0.05^{*}$ \\
\cline { 2 - 6 } & Study & $0.375 \pm 0.013$ & $0.509 \pm 0.0194$ & 27.38 & $<0.05^{*}$ \\
\hline \multirow{2}{*}{ Spine } & Control & $0.460 \pm 0.018$ & $0.51 \pm 0.012$ & 9.79 & $<0.05^{*}$ \\
\cline { 2 - 5 } & Study & $0.455 \pm 0.018$ & $0.594 \pm 0.036$ & 13.92 & $<0.05^{*}$ \\
\hline
\end{tabular}

SD: standard deviation, Post: post- treatment, p- value: probability value, ${ }^{*}$ : significant difference, t-value: paired t- test

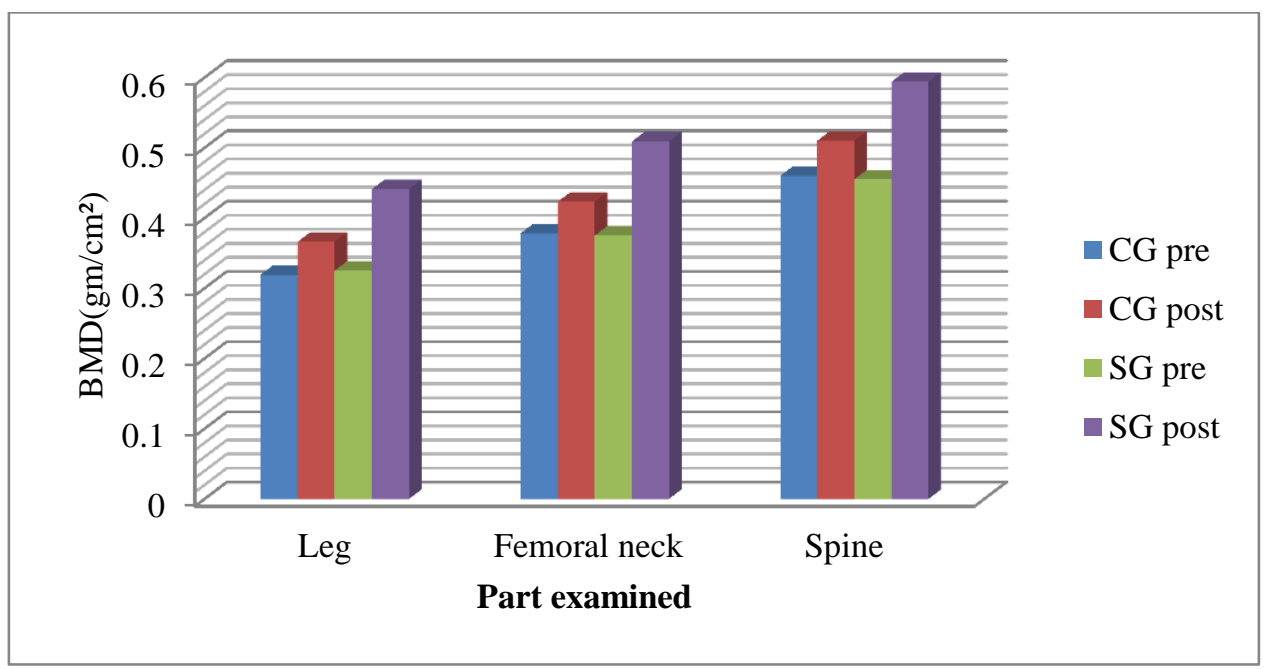

CG pre: control group pre-treatment

CG post: control group post-treatment
SG pre: study group pre- treatment

SG post: study group post- treatment

Figure 2: Pre and post- treatment mean values of measured variables within control and study group 


\section{Discussion}

The results of this study showed that 12 weeks of WBV resulted in increase in BMD of the leg ,the femoral neck and the spine in children with MMC .Choosing DEXA in this study because it is the gold standard for the diagnosis of osteoporosis, the most commonly used method for measuring BMD ,because of its recognized advantages in clinical use; specifically, exposure to very low doses of ionizing radiation, good precision, short scan times and stable calibration. DEXA equipment allowed us to scan the spine and hip, which are considered as the most important measurement sites. This is because the predictive ability of BMD for fracture is site-specific, and the spine and hip are common sites of osteoporotic fractures associated with substantial impairment of quality of life, and increased morbidity and mortality. A measurement of hip BMD has been shown to be the most reliable way of evaluating the risk of hip fracture $[18,19]$.

Comparison of the pre-treatment results of both control and study groups with regard to all the measured variables revealed no significant difference $(\mathrm{P}>0.05)$, suggesting that both groups were homogenous at the beginning of the study. Comparison of post treatment mean values of femoral neck, spine and legs BMD revealed more improvement in favor of the study group $(\mathrm{P}<0.05)$. The mechanism(s) by which low-level mechanical vibration can enhance the musculoskeletal system are currently unknown [20]. The physical basis of translating lowlevel mechanical signals into a biological response could result from an amplification system achieved through fluid movement through the canalicular system of osteocytes [21] and enhanced by the interdependence of fluid pressure and frequency [22].

From a biologic viewpoint, the improvement in bone mass could result from alterations in the transcriptional control of the bone tissue either by up regulating genes responsible for bone formation, down regulating genes involved in the resorption of bone, or both [23]. Certainly, it is possible that adaptation of the musculoskeletal system to exogenous signals is preferentially sensitive to higher frequency signals, similar to other physiologic systems designed to monitor "exogenous stimuli," such as hearing (tone) ,vision (color), and tactile sense (pressure), and that these external signals are processed within specific windows of sensitivity and begin to shut down when the signal becomes too bright, too loud, or too heavy. The physical and biologic mechanisms that control the adaptation of bone to its loading environment are complex [24] and involve the interaction of pathways mediated through muscle contractions, gravity, and physical activity, as well as a genetic factor that defines the musculoskeletal system's susceptibility to mechanical signals [25]. Whereas the strain signals in this study fell well below those that are imposed on the skeleton by vigorous exercise [26], they were significantly more strong than those practiced during minimal activities of daily life [27].

Our results come in agreement with Lam et al.[28] who conducted a study to determine whether WBV therapy was useful for treating osteopenia in adolescent idiopathic scoliosis patients. Results showed that WBV was effective for improving areal bone mineral density at the femoral neck of the dominant side and lumbar spine. Also, our results come in agreement with Ruck et al.[29]who studied the effects of WBV on BMD in cerebral palsy children. The study group received their regular physiotherapy treatments plus WBV, whereas the control group only underwent the regular physical therapy. The results showed significant increase in BMD of the distal femur metaphysis in the WBV group only.

\section{Conclusion}

The importance of this study comes from that it provided evidence that WBV can be used as an additional method to increase BMD of children with MMC without exposing them to fractures that may happen during vigorous stretch during traditional physical therapy. This study included 20 children which may limit the generalization of the statistical findings. On the basis of our findings, we advise the use of WBV on different age groups of children with MMC. Further research should be done to investigate the effect of increasing period of treatment on bone mineral density in these children. Further studies are recommended to detect the effect of WBV on motor development of these children. Further researches are needed to determine various factors affecting bone density in children with MMC. Comparative studies are needed to investigate the effect of WBV on different types of pediatric disorders. The data from this study indicate that the formation of bone can be enhanced in children with MMC by short exposure to low-magnitude high frequency mechanical vibration.

\section{Acknowledgement}

The author would like to express his appreciation to all the children and their parents for their co-operation and participation in this study.

\section{Conflict of interest}

No potential conflict of interest reveal to this article was reported. 


\section{References}

[1] S.L. Boulet, Q.Yang, C .Mai et al., Trends in the postfortification prevalence of spina bifida and anencephaly in the United States, Birth Defects Res A Clin Mol Te ratol,82,2008,527-532.

[2] L.E. Mitchell, N.S. Adzick, J. Melchionne, et al., Spina bifida, Lancet ,364,2004,1885-1895.

[3] WHO Scientific Group on the Prevention and Management of Osteoporosis. Prevention and management of osteoporosis: report of a WHO scientific group, Internet Communication, 2007.

[4] K.M.Valtonen, L.A.Goksor, O.Jonsson, D.Mellstrom, H.T. Alaranta and E.R. Viikari-Juntura , Osteoprosis in adults with meningomyelocele, an unrecognized problem at rehabilitation clinics,Arch. Phys. Med. Rehabil,87(3),2006, 376-82-305.

[5] K.Parsch, Origin and treatment of fractures in spina bifida,Eur.J. Pediatr. Surg, 1(5)1991, 298-306.

[6] D.G.Dolny, and G.F.C. Reyes,Whole body vibration exercise: training and benefits,Curr. Sports Med. Rep., 7( 3),2008, $152-157$.

[7] C .Rubin, R. Recker, D .Cullen, et al. ,Prevention of postmenopausal bone loss by a low-magnitude, high-frequency mechanical stimuli:a clinical trial assessing compliance, efficacy, and safety,J Bone Miner Res, 19, 2004,343-351.

[8] C .Rubin, A.S.Turner, S .Bain, et al. ,Anabolism. Low mechanical signals strengthen long bones,Nature,412,2001,603-604.

[9] S .Torvinen, P .Kannus, H. Sievanen, et al., Effect of 8-month vertical whole body vibration on bone, muscle performance, and body balance:a randomized controlled study,J BoneMiner Res. ,18, 2003,876-884.

[10] K .Ward, C .Alsop, J .Caulton, et al. Low magnitude mechanical loading is osteogenic in children with disabling conditions, J Bone Miner Res., 19,2004,360-369.

[11] V. Gilsanz, T.A. Wren, M. Sanchez, et al., Low-level, high-frequency mechanical signals enhance musculoskeletal development of young women with low BMD,J Bone Miner Res., 21,2006,1464-1474.

[12] C .Bosco, R .Colli, E .Introini, et al. ,Adaptive responses of human skeletal muscle to vibration exposure. Clin Physiol,19, 1999, 183187.

[13] C .Rubin, G. Xu and S.Judex, The anabolic activity of bone tissue,suppressed by disuse, is normalized by brief exposure to ex tremely low-magnitude mechanical stimuli, Faseb J., 15,2001,2225-2229.

[14] C.T.Rubin, D.W. Sommerfeldt, S. Judex, et al., Inhibition of osteopenia by low magnitude, high-frequency mechanical stimuli,Drug Discov Today, 6, 2001,848-858.

[15] N .Carter, E .Duncan and P.Wordsworth, Bone mineral density in adults with Marfan syndrome,Rheumatology, 39,2000,307-309.

[16] O .Semler, O. Fricke, K.Vezyroglou, C .Stark, E. Schoenau,Preliminary results on the mobility after whole body vibration in immobilized children and adolescents, J Musculoskelet Neuronal Interact,7,2007,77-81.

[17] O.Semler, O. Fricke, K. Vezyroglou, C. Stark, A. Stabreyand E. Schoenau, Results of a prospective pilot trial on mobility after whole body vibration in children and adolescents with osteogenesis imperfect,Clin Rehab,22,2008,387-94.

[18] D. Marshall, O. Johnell and $\mathrm{H}$.Wedel, Meta-analysis of how well measures of bone mineral density predict occurrence of osteoporotic fractures,BMJ, 1996, 312, 1254-59.

[19] S.R. Cummings, D.M .Black, M.C. Nevitt, W .Browner, J .Cauley, K .Ensrud et al. ,Bone density at various sites for prediction of hip fractures, Lancet,341, 1993,72-75.

[20] J. Rubin, C. Rubin and C.R. Jacobs , Molecular pathways mediating mechanical signaling in bone, Gene, 367,2006,1-16.

[21] L.Wang, S.P. Fritton, S.C. Cowin and S. Weinbaum ,Fluid pressure relaxation depends upon osteonal microstructure: Modeling an oscillatory bending experiment,J Biomech, 32,1999,663-672.

[22] Y.X.Qin,T. Kaplan, A .Saldanha and C. Rubin, Fluid pressure gradients, arising from oscillations in intramedullary pressure,is correlated with the formation of bone and inhibition of intracortical porosity,J Biomech 36,2003,1427-1437.

[23] S .Judex, N .Zhong, M.E. Squire, K. Ye, L.R .Donahue, M .Hadjiargyrou and C.T. Rubin , Mechanical modulation of molecular signals which regulate anabolic and catabolic activity in bone tissue,J Cell Biochem ,94,2005,982-994.

[24] G.Karsenty ,The complexities of skeletal biology. Nature 423,2003,316-318.

[25] S.Judex,R.Garman ,M. Squire, L.R.Donahue and C. Rubin ,Genetically based influences on the site-specific regulation of trabecular and cortical bone morphology, J Bone Miner Res,19,2004,600-606.

[26] C.T. Rubin and L.E. Lanyon, Dynamic strain similarity in vertebrates; an alternative to allometric limb bone scaling,J Theor Biol ,107,1984,321-327.

[27] S.P.Fritton, K.J.McLeod and C.T.Rubin, Quantifying the strain history of bone: Spatial uniformity and self- similarity of low magnitude strains , J Biomech,33,2000,317-325.

[28] T. P.Lam, B. K. W . Ng, L. W. H. Cheung, M. K.Lee, L. Qin and J. C. Y. Cheng, Effect of whole body vibration (WBV) therapy on bone density and bone quality in osteopenic girls with adolescent idiopathic scoliosis: a randomized, controlled trial, Osteoporosis International ,24( 5),2013, 1623-1636.

[29] J.Ruck, G.Chabot, F.Rauch, Vibration treatment in cerebral palsy: a randomized controlled pilot study. J Musculoskel Neur Interact, $10(1), 2010,77-83$. 